\section{Small Broad-Host-Range lacZ Operon Fusion Vector with Low Background Activity}

BioTechniques 31:1258-1262 (December 2001)

The use of gene fusion technologies for transcriptional analysis of gene expression, especially of those genes whose products are difficult to assay, has been well documented (12-15). Many narrow and broad-host-range plasmid-based systems are available for constructing such gene fusions $(6,7$, 14). To circumvent some of the inherent problems of plasmid-based systems, which have been discussed before (3), systems have been developed for single-copy, chromosomal insertion of gene fusions $(4,9,14)$. For some applications (e.g., deletion mapping of regulatory regions or use in bacteria for which no integration-proficient vectors are known), plasmid-based fusions are still indispensable. The most useful vectors use a promoterless $\beta$-galactosidase $(\beta$-gal) gene, lac $Z$, as a reporter. However, most broad-host-range $l a c Z$ based vectors available to date exhibit some potentially serious drawbacks, including (i) high levels of background expression due to transcription from plasmid-encoded promoters and/or effi cient translation of low levels of lacZ message due to a strong consensus Shine-Dalgarno (SD) sequence, (ii) a lack of unique cloning sites, and (iii) large size due to the presence of unknown and usually unnecessary nucleotide sequences. To address these is sues, a new small broad-host-range transcriptional lac $Z$ operon fusion vector was constructed and tested in Pseudomonas aeruginosa.

The new lacZ vector, pTZ110 (Figure 1A), was obtained in two steps. First, a 1544-bp blunt-ended Pst I-StuI fragment from pSF2 (10), containing an origin of transfer, the ori 1600 broadhost-range origin of replication and its associated replication protein (16), was ligated into a BalI site located $271 \mathrm{nu}-$ cleotides downstream of the lac $Z$ gene of pGE593 (2) to obtain pTZ100. This operon fusion vector contains unique

A)

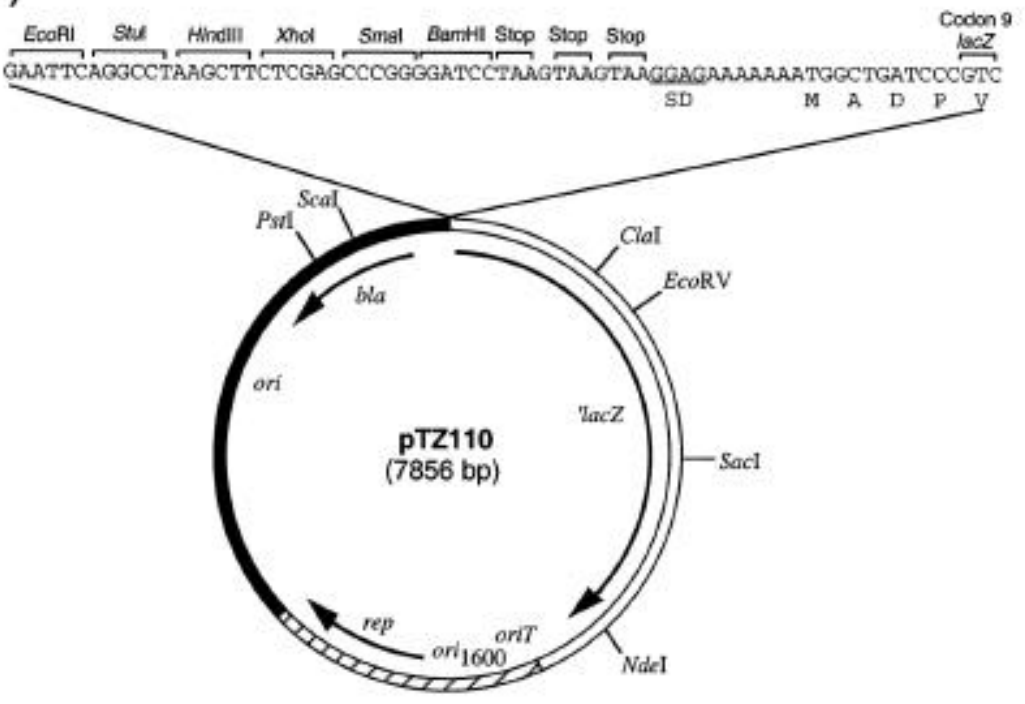

B)

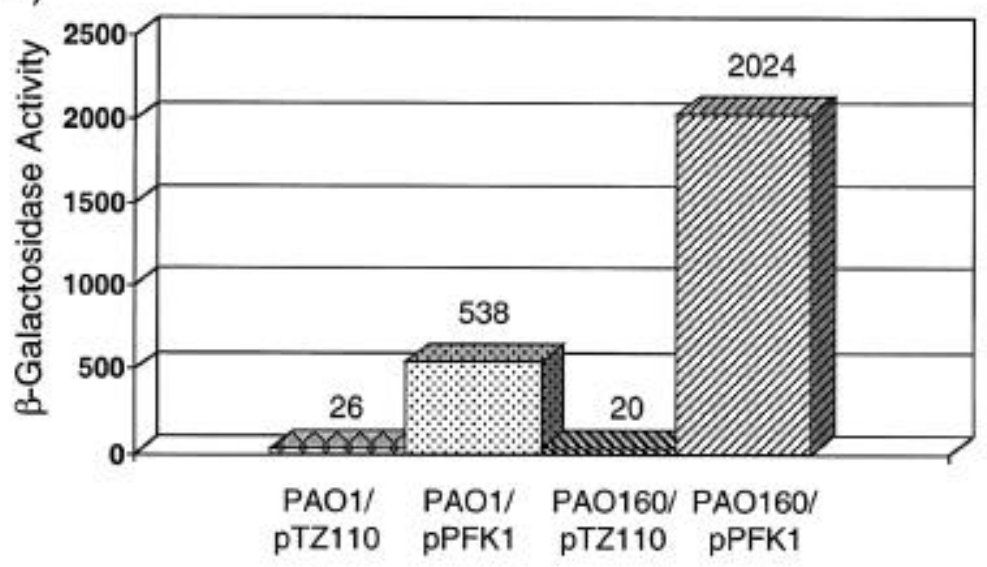

Figure 1. Map of the operon fusion vector pTZ110 (A) and GlpR-dependent glpF-lacZ expression in $\boldsymbol{P}$. aeruginosa (B). (A) The sequence preceding the promoterless lacZ ('lacZ) gene contains stop codons in all frames and an SD sequence that is close to consensus but not too strong. Other abbreviations: bla, $\beta$-lactamase-encoding gene; ori, pBR322 origin of replication; ori 1600 , pRO1600 origin of replication; oriT, origin of transfer; rep, ori $i_{1600}$ replication protein. The sequence of pTZ110 was submitted to GenBank ${ }^{\circledR}$ and assigned accession no. AF376056. (B) Transcriptional activity of the $g l p F K$ operon promoter present on pPFK1 was assessed by measuring either $\beta$-gal activities in strains PAO1 (wild-type) or PAO160 $(g l p R)$ containing the indicated plasmids. The strains were grown aerobically at $37^{\circ} \mathrm{C}$ in LB medium (Invitrogen, Carlsbad, CA, USA) to log phase (absorbance at $540 \mathrm{~nm}$ of approximately 0.7$)$. $\beta$-gal expression was measured in chloroform/SDS-permeabilized cells, and activity units were calculated as previously described (5). Samples were assayed in triplicate, and the average values are given above the columns.

EcoRI, SmaI, and BamHI sites upstream of a promoterless lacZ gene. Second, pTZ110 was obtained by ligating a 26-bp oligonucleotide linker with EcoRI and BamHI overhangs between the same sites of pTZ100. The resulting operon fusion vector contains a multiple cloning site with six unique restriction enzyme cleavage sites, stop codons in the three reading frames, and a modified SD sequence, which is close to consensus but not too strong. The multiple cloning site was designed to contain blunt-end generating sites close to each end and compatible sites (e.g., Bam HI and XhoI allow cloning of fragments generated with $B g l \mathrm{II}$ and SalI, respectively). Using the pBR322-based repli- 
con, this vector replicates with an intermediate copy number in E. coli and using ori $_{1600}$ with a low copy number in Pseudomonas (10-15 copies) (8). These copy numbers allow for easy isolation and manipulation of plasmid DNA from E. coli hosts, while they are at the same time desirably low for analysis of gene expression in Pseudomonas hosts.

To assess the functionality of pTZ110, a glpF-lacZ transcriptional fusion was constructed and analyzed. The glpF gene is part of the $P$. aeruginosa glpFK operon, whose expression is negatively regulated by the glycerol-3phosphate regulon repressor, GlpR (11). The amino-terminal 14 codons of $g l p F$ and its upstream 219-bp regulatory region containing a single GlpR operator site were PCR amplified from pPS585 (11). The reactions contained the 24-mer universal lac $Z$ primer and a $g l p F$-specific primer ( $5^{\prime}$-CTCGGAC $\underline{\text { A- }}$ GGCcTTGGCCGAACAG-3'), which introduced a single base change (lowercase letter) and therefore a StuI site (underlined) at codon 14 of $g l p F$. The resulting PCR fragment was digested with EcoRI and StuI, and a 282-bp EcoRI-StuI fragment was gel-purified and ligated between the EcoRI and SmaI sites of pTZ110 to form pPFK1. In this construct, translation of an 18amino-acid GlpF fusion peptide is terminated at the second TAA codon provided by pTZ110. The correct physiological response pattern of the $g l p F$ lac Z fusion was assessed by conjugating pPFK1 from E. coli strain SM10 into wild-type $P$. aeruginosa PAO1 and the PAO1 glpR mutant PAO160 (11). Exconjugants were selected on minimal citrate medium (3) containing 100 $\mu \mathrm{g} / \mathrm{mL}$ carbenicillin. Alternatively, plasmids were transferred by electroporation (1) followed by selection on LB medium containing $100 \mu \mathrm{g} / \mathrm{mL}$ carbenicillin. $\beta$-gal expression from the glpF-lac Z operon fusion was then measured after growth in the appropriate media (Figure 1B). As expected, $g l p F$ lac $Z$ was expressed in a GlpR-dependent manner, and very low background $\beta$-gal activity was observed in the pTZ110-containing strains. $\beta$-gal expression in the wild-type strain is not completely repressed because LB medium contains traces of the inducer glycerol-3-phosphate and glpFK operon ex- pression is easily induced by dislodging GlpR from the single operator site (11).

In summary, with the construction of the pTZ110 lac $Z$ operon fusion vector, several problems associated with similar broad-host-range fusion vectors have been resolved. First, background $\beta$-gal expression levels are minimized because of the incorporation of an SD sequence that is close to consensus but not too strong. Second, cloning of promoter-containing fragments is facilitated by six unique cloning sites, including two blunt-end-generating restriction sites. Third, the complete nucleotide sequence of the new small 7856-bp vector is known, and it contains only fully functional DNA sequences. Although this broad-hostrange vector has thus far only been tested in $P$. aeruginosa, it will undoubtedly be useful with the many Pseudomonads and other bacteria in which the ori $i_{1600}$ replicon is functional (10).

\section{REFERENCES}

1.Dennis, J.J. and P.A. Sokol. 1995. Electrotransformation of Pseudomonas. In J.A. Nickoloff (Ed.), Methods in Molecular Biology, Electroporation Protocols for Microorganisms. Humana Press, Totowa, NJ.

2.Eraso, J.M. and G.M. Weinstock. 1992 Anaerobic control of Colicin E1 production. J. Bacteriol. 174:5101-5109.

3.Hoang, T.T., A.J. Kutchma, A. Becher, and H.P. Schweizer. 2000. Integration proficient plasmids for Pseudomonas aeruginosa: sitespecific integration and use for engineering of reporter and expression strains. Plasmid 43:59-72.

4.Linn, T. and R. St. Pierre. 1990. Improved vectors system for constructing transcriptional fusions that ensures independent translation of lacZ. J. Bacteriol. 172:1077-1084.

5.Miller, J.H. 1992. A Short Course in Bacterial Genetics. CSH Laboratory Press, Cold Spring Harbor, NY.

6.Ronald, S.L., A.M. Kropinski, and M.A. Farinha. 1990. Construction of broad-hostrange vectors for the selection of divergent promoters. Gene 90:145-148.

7.Santos, P.M., I. Di Bartolo, J.M. Blatny, E. Zennaro, and S. Valla. 2001. New broadhost-range promoter probe vectors based on the plasmid RK2 replicon. FEMS Microbiol. Lett. 195:91-96.

8.Schweizer, H.P. 1991. Escherichia-Pseudomonas shuttle vectors derived from pUC18/19. Gene 97:109-112.

9.Schweizer, H.P. and A. Becher. 2000. Integration-proficient Pseudomonas aeruginosa vectors for isolation of single copy chromosomal $l a c Z$ and $l u x$ gene fusions. BioTechniques 29:948-954.
10.Schweizer, H.P., T.R. Klassen, and T. Hoang. 1996. Improved methods for gene analysis and expression in Pseudomonas, $\mathrm{p}$. 229-237. In T. Nakazawa, K. Furukawa, D. Haas, and S. Silver (Eds.), Molecular Biology of Pseudomonads. American Society for Microbiology, Washington, D.C.

11.Schweizer, H.P., C. Po, and R. Jump. 1997. Structure and gene-polypeptide relationships of the region encoding glycerol diffusion facilitator $(g l p F)$ and glycerol kinase $(g l p K)$ of Pseudomonas aeruginosa. Microbiology 143:1287-1297.

12.Shuman, H.A., T.J. Silhavy, and J.R. Beckwith. 1980. Labeling of proteins with $\beta$-galactosidase by gene fusion: identification of a cytoplasmic membrane component of the Escherichia coli maltose transport system. J. Biol. Chem. 255:168-174.

13.Silhavy, T.J. and J.R. Beckwith. 1985. Uses of lac fusions for the study of biological problems. Microbiol. Rev. 49:398-418.

14.Simons, R.W., F. Houman, and N. Kleckner. 1987. Improved single and multicopy lacbased cloning vectors for protein and operon fusions. Gene 53:85-96.

15.Slauch, J.M. and T.J. Silhavy. 1991. Genetic fusions as experimental tools. Methods Enzymol. 204:213-248.

16.West, S.E.H., H.P. Schweizer, C. Dall, A.K. Sample, and L.J. Runyen-Janecky. 1994. Construction of improved EscherichiaPseudomonas shuttle vectors derived from pUC18/19 and the sequence of the region required for their replication in Pseudomonas aeruginosa. Gene 128:81-86.

Work in the authors' laboratory is supported in part by National Institutes of Health grant no. GM56685. Address correspondence to Dr. Herbert P. Schweizer, Department of Microbiology, Colorado State University, Fort Collins, CO 805231677, USA. e-mail: herbert.schweizer@ colostate.edu

Received 9 July 2001; accepted 14 August 2001.

\section{Herbert P. Schweizer and Rungtip Chuanchuen Colorado State University Fort Collins, CO, USA}

